\title{
The First Detection of Cobalt In A Damped Lyman Alpha System
}

\author{
S. L. Ellison ${ }^{1}$, S. G. Ryan ${ }^{2}$, J. X. Prochaska ${ }^{3}$ \\ ${ }^{1}$ European Southern Observatory, Casilla 19001, Santiago 19, Chile \\ ${ }^{2}$ Dept of Physics \& Astronomy, The Open University, Walton Hall, Milton Keynes, MK7 6AA, UK \\ ${ }^{3}$ Carnegie Observatories, Pasadena, CA 91101, USA
}

\begin{abstract}
The study of elemental abundances in Damped Lyman Alpha systems (DLAs) at high redshift represents one of our best opportunities to probe galaxy formation and chemical evolution at early times. By coupling measurements made in high $z$ DLAs with our knowledge of abundances determined locally and with nucleosynthetic models, we can start to piece together the star formation histories of these galaxies. Here, we discuss the clues to galactic chemical evolution that may be gleaned from studying the abundance of Co in DLAs. We present high resolution echelle spectra of two QSOs, Q2206-199 and Q1223+17, both already known to exhibit intervening damped systems. These observations have resulted in the first ever detection of Co at high redshift, associated with the $z_{a b s}=1.92 \mathrm{DLA}$ in the sightline towards Q2206-199. We find that the abundance of $\mathrm{Co}$ is approximately $\frac{1}{4} Z_{\odot}$ and that there is a clear overabundance relative to iron, $[\mathrm{Co} / \mathrm{Fe}]=+0.31 \pm 0.05$. From the abundance of $\mathrm{Zn}$, we determine that this is a relatively metal-rich DLA, with a metallicity approximately $\frac{1}{3} Z_{\odot}$. Therefore, this first detection of $\mathrm{Co}$ is similar to the marked overabundance relative to $\mathrm{Fe}$ seen in Galactic bulge and thick disk stars.
\end{abstract}

Key words: Quasars - absorption lines: ISM - abundances: galaxies - evolution

\section{INTRODUCTION}

Our understanding of the processes associated with galactic chemical enrichment, such as stellar nucleosynthesis, dust depletion and mixing mechanisms, can benefit greatly from the comparison of local abundances with those measured at high redshift. At low $z$, abundances can be determined for the Milky Way and its near neighbours from stars, H II regions and the interstellar medium (e.g. Chen et al. 1999; Kobulnicky and Zaritsky 1999; Shetrone et al. 2000; Izotov and Thuan 1999; Savage and Sembach 1996). At high redshift, the most accurate and extensive abundance measurements are those determined from Damped Lyman Alpha systems (DLAs). These systems are the highest H I column density members of the quasar absorption line menagerie and are traditionally defined as having $\mathrm{N}(\mathrm{H} \mathrm{I}) \geq 2 \times 10^{20}$ atoms $\mathrm{cm}^{-2}$. Coupling measurements of metal abundances in high redshift DLAs with our knowledge of local enrichment patterns plays a crucial role in our understanding of the star formation histories of these distant objects and the early stages in galaxy evolution.

In the context of investigating galactic chemical evolution, several key elements have previously been targeted as particularly informative diagnostics. The utility of these key metals is often based on our knowledge of their relative production and release timescales in stars (and subsequent supernovae) of different masses. For example, the $\alpha$ elements ( $\mathrm{Mg}, \mathrm{Si}, \mathrm{Ca}$ etc.) have been extensively used to investigate the amount and rate of massive star formation. An overabundance of $\alpha$ relative to Fe in metal-poor Galactic halo stars chronicles the epoch of early star formation (Tinsley 1979; Ryan, Norris and Beers 1996) and in the bulge the same overabundances in solar metallicity stars are indicative of a 'get rich quick' population (McWilliam and Rich 1994; Rich and McWilliam 2000) i.e. one which is as old as the Galactic halo but as metal-rich as the Galactic disk. In DLAs, there is on-going discussion about the abundances of $\alpha$ elements, for which nucleosynthetic origins of abundance patterns must be disentangled from dust depletion effects. Although there is some evidence that Si may be overabundant in DLAs (Lu, Sargent and Barlow 1998), [Si/Zn] is solar in at least some systems (Pettini et al 2000). Moreover, since $\mathrm{Si}$ is highly refractory, the interpretation of [Si/Zn] measurements relies on a dust correction factor based on $[\mathrm{Zn} / \mathrm{Cr}]$, which is quite uncertain. Conversely, the abundance of $\mathrm{S}$, an $\alpha$ element not depleted onto dust, has been found by Centurion et al. (2000) to be underabundant relative to Fe, a result at odds with the Type II supernova pattern observed in the Milky Way. These results have been interpreted as possible evidence for star formation having occurred at a

(C) 2001 RAS 
low rate or in sporadic bursts. However, the Centurion et al. (2000) data were obtained with resolution $\mathrm{R}=5000$ and need to be followed up with higher resolution data in order reliabley distinguish the $\mathrm{S}$ lines from the Lyman $\alpha$ forest. Further clues to the star formation history of DLAs may be gleaned from N/O ratios. The large observed spread in $\mathrm{N} / \mathrm{O}$ in DLAs can be explained by the delayed release of primary $\mathrm{N}$ (relative to $\mathrm{O}$ ) in metal-poor intermediate mass stars (Pettini et al. 2001; Lattanzio et al. 2001).

Here we focus on cobalt, an element which although relatively widely studied in Galactic stars, has not yet been featured in DLA abundance studies. The intriguing trends of $[\mathrm{Co} / \mathrm{Fe}]$ as a function of $[\mathrm{Fe} / \mathrm{H}]$ in the various Galactic stellar populations indicate that cobalt may be another element that can provide important clues to chemical evolution. In sub-solar metallicity thin disk stars, Co is found to be slightly underabundant with respect to $\mathrm{Fe}([\mathrm{Co} / \mathrm{Fe}]$ $\sim-0.1$, Gratton and Sneden 1991), although this may be related to their solar $\log g f$ scale being on average $0.06 \pm 0.04$ higher than the laboratory scale adopted by many other observers (see Section 5.1 for more details). This trend continues in moderately metal-poor halo stars down to metallicities of $[\mathrm{Fe} / \mathrm{H}] \sim-2.5$. For more metal-poor halo stars, a considerable overabundance of Co with respect to $\mathrm{Fe}$ is observed, $0<[\mathrm{Co} / \mathrm{Fe}]<0.8$, (McWilliam et al. 1995; Ryan, Norris and Beers 1996). Interestingly, this coincides with a downturn in relative $\mathrm{Cr}$ to $\mathrm{Fe}$ abundances, such that Co and $\mathrm{Cr}$ are closely anti-correlated in the metal poor population. Similar $[\mathrm{Co} / \mathrm{Fe}]$ overabundances are observed in the bulge ([Co/Fe $] \sim+0.3$, McWilliam \& Rich 1994), which, we recall, is as old as the halo, and to a lesser degree in the thick disk $([\mathrm{Co} / \mathrm{Fe}] \sim+0.1$, Prochaska et al. 2000).

The detection of Co II transitions is a challenging possibility for ISM absorption line spectroscopy, both locally and at high $z$. Although there are numerous transitions that lie at convenient rest wavelengths for both local and high redshift studies, only two interstellar measurements currently exist. These detections have been achieved in the local ISM in the sightlines towards $\zeta$ Oph (Federman et al. 1993) and $\rho$ Oph A (Mullman 1998b) using the Co II $\lambda 1466$ transition which has an equivalent width in these interstellar clouds of only 0.5 and $4.7 \mathrm{~m} \AA$ respectively. One of the advantages of moving to higher redshifts is that the relatively strong UV lines become shifted into the optical where one has access to more efficient detectors and larger telescopes. In addition, the observed equivalent width is increased by a factor of $(z+1)$. However, the fundamental problem remains that the Co II lines are all intrinsically weak and that the solar abundance of Co is $8 \times 10^{-8}$ that of hydrogen. In addition, Mullman et al. (1998a,b) have provided new laboratory measurements for Co II $f$-values (oscillator strengths), typically revising the old values downwards by a factor of approximately two. This means that the spectral detection limit has effectively been increased by the same factor. Finally, the two local detections have brought to light an additional problem, namely that cobalt appears to be a highly refractory element that is depleted onto dust to a similarly high degree as iron (Mullman et al. 1998b).

Despite the technical challenge of observing Co in the high redshift ISM of DLAs, it is now feasible with high resolution spectrographs on $8-10 \mathrm{~m}$ class telescopes to detect this element, in reasonable exposure times, down to log
$N(\mathrm{Co}) \sim 12.5$. Here we present the first ever detection of Co in a DLA, at $z_{\text {abs }}=1.92$, determined from a spectrum obtained with the Ultra-violet and Visual Echelle Spectrograph (UVES) on UT2 of the VLT. In addition, we present a combined UVES and HIRES (High Resolution Echelle Spectrograph) spectrum obtained at the VLT and Keck telescopes respectively, which can also offer an interesting upper limit to $[\mathrm{Co} / \mathrm{H}]$ in a second DLA. We discuss the implications for the star formation history of these high redshift galaxies and compare our observations with recent nucleosynthetic yield models.

\section{TARGET SELECTION}

In order to at least place interesting limits on the abundance of Co in DLAs, it is desirable to reach a sensitivity level of $[\mathrm{Co} / \mathrm{Fe}]=0$. Such a detection limit is sufficient at least to distinguish between the marked overabundance of Co relative to Fe seen in the halo, bulge, and to a lesser degree in the thick disk, and the mild underabundance in the Galactic thin disk. Therefore, in order to maximise the likelihood of a Co II detection, we have selected bright QSOs with foreground DLAs known to contain high column densities of Fe. This criterion can either be achieved in moderate metallicity DLAs with high $N(\mathrm{H} \mathrm{I})$ or in lower column density systems with relatively high levels of enrichment. The former type of system is relatively rare due to the power law distribution of column densities in $\mathrm{H}$ I absorbers which shows that systems with $N(\mathrm{H} \mathrm{I}) \gtrsim 2 \times 10^{21} \mathrm{~cm}^{-2}$ are very scarce. Similarly, few metal rich $\left(Z>\frac{1}{10} Z_{\odot}\right)$ DLAs have been identified at any redshift (Pettini et al. 1999), indicating that extinction due to dust may be biasing current samples (Ellison et al. 2000). Finally, as dust extinction may be greater in metalrich and high- $N(\mathrm{H})$ DLAs (e.g. Prantzos and Boissier 2000), it is particularly challenging to identify QSOs absorbed by such systems that also are bright enough to provide the requisite $\mathrm{S} / \mathrm{N}$.

Having searched the literature for candidate targets, we initially identified two QSOs for study, Q2206-199 ( $B=$ $\left.17.8, z_{e m}=2.559\right)$ and Q1223+17 $\left(B=18.5, z_{e m}=2.936\right)$. The former of these targets actually exhibits 3 separate DLAs in its absorption spectrum (Prochaska and Wolfe 1997); here we focus on the system at $z_{a b s}=1.92$. Although this system only has a moderate column density compared with other DLAs $\left(N(\mathrm{H} \mathrm{I})=4.8 \times 10^{20} \mathrm{~cm}^{-2}\right.$, see section $4)$, it is amongst the most metal-rich systems known with $Z \sim \frac{1}{3} Z_{\odot}$. In fact, Prochaska and Wolfe (1997) were able to detect some elements rarely seen in DLAs in this system, such as Ti. Conversely, Q1223+17 is a very high column density absorber, $N(\mathrm{H} \mathrm{I})=3 \times 10^{21} \mathrm{~cm}^{-2}$ and therefore has a relatively high column density of Fe despite its low metallicity (Pettini et al. 1994; Prochaska et al. 2001).

\section{OBSERVATIONS}

We present high resolution echelle spectra for these two high redshift QSOs. The data were obtained with UVES on the VLT (Q2206-199 and Q1223+17) and with HIRES on the Keck telescope (Q1223+17). A summary of the observations is given in Table 1 . 
Table 1. Summary of Observations

\begin{tabular}{llllrr}
\hline \hline Quasar & Telescope/Instrument & Max. $\lambda$ Coverage & Resolution & Int Time $(\mathrm{s})$ & $\mathrm{S} / \mathrm{N}$ \\
\hline Q2206-199 & VLT/UVES & $3300-6650 \AA$ & 42000 & 17100 & $20-70$ \\
Q1223+17 & VLT/UVES & $3800-10250 \AA$ & 45000 & 10800 & $20-50$ \\
Q1223+17 & Keck/HIRES & $4750-7200 \AA$ & 38000 & 19100 & $\sim 30$ \\
\hline
\end{tabular}

\subsection{Details of Observations and Data Reduction of Q2206-199}

Spectra of Q2206-199 were obtained over 3 nights from May 28-30 2000 with UVES on the VLT in conditions of good seeing, typically $0.5-0.7$ arcseconds, although for brief periods extremes of 0.4 and 1.3 arcsecs were reached. A slit width of 1.0 arcsec was fixed throughout the observations, oriented along the parallactic angle. The data presented here were obtained with the dichroic $1 \lambda_{\text {cen }}=390+564$ configuration and read out in $2 \times 2$ pixel-binned, high-gain mode. The blue arm CCD is a single thinned EEV chip whereas the red arm consists of a mosaic of a second EEV chip and an MIT chip, separated by approximately $0.96 \mathrm{~mm}$. A single dichroic setting therefore has non-contiguous wavelength coverage and in the $\lambda_{\text {cen }}=390+564$ setting there are gaps between $4525-4625$ and $5600-5680 \AA$.

The extraction of the spectra was achieved using a customized version of the UVES pipeline which is based on ECHELLE routines in the data reduction package MIDAS. A detailed description of this process can be found in Ballester et al. (2000). The spectra were optimally extracted separately for the three CCDs and the variation in the continuum flux level, due primarily to the blaze function of the echelle grating, was removed. The resolution of the spectra, as determined from ThAr arc lines, is $R \sim 43000$, or approximately $7 \mathrm{~km} \mathrm{~s}^{-1}$ FWHM. 1-D spectra were obtained by joining the orders and converting the wavelengths to a vacuum heliocentric scale. The individual spectra were then co-added with a weighting proportional to their S/N. The final step was to normalise the spectrum by dividing through by a spline function fitted through absorption-free regions of data. Since most of the metal lines which we study here are found redward of the Lyman $\alpha$ emission, the continuum can be accurately fitted since there are relatively few absorption features and the $\mathrm{S} / \mathrm{N}$ is quite high.

\subsection{Details of Observations and Data Reduction of $\mathrm{Q} 1223+17$}

The spectrum presented here of the $z_{a b s}=2.466$ DLA towards Q1223+17 is a composite of HIRES plus UVES data. The UVES data were obtained during the same run as the Q2206-199 spectra and reduced in an identical way. The only difference for the data of Q1223+17 is that they were obtained with the dichroic $2 \lambda_{\text {cen }}=437+860$ setting. This mode offers a larger, redder overall wavelength coverage at the expense of slightly larger inter-arm gaps (between 5000 - $6750 \AA$ and $8500-8750 \AA$ ). In addition to the UVES data, we have obtained $\sim 5$ hours of Keck HIRES data, which are described in more detail by the comprehensive abundance analysis of Prochaska et al. (2001). In brief, the C5 decker
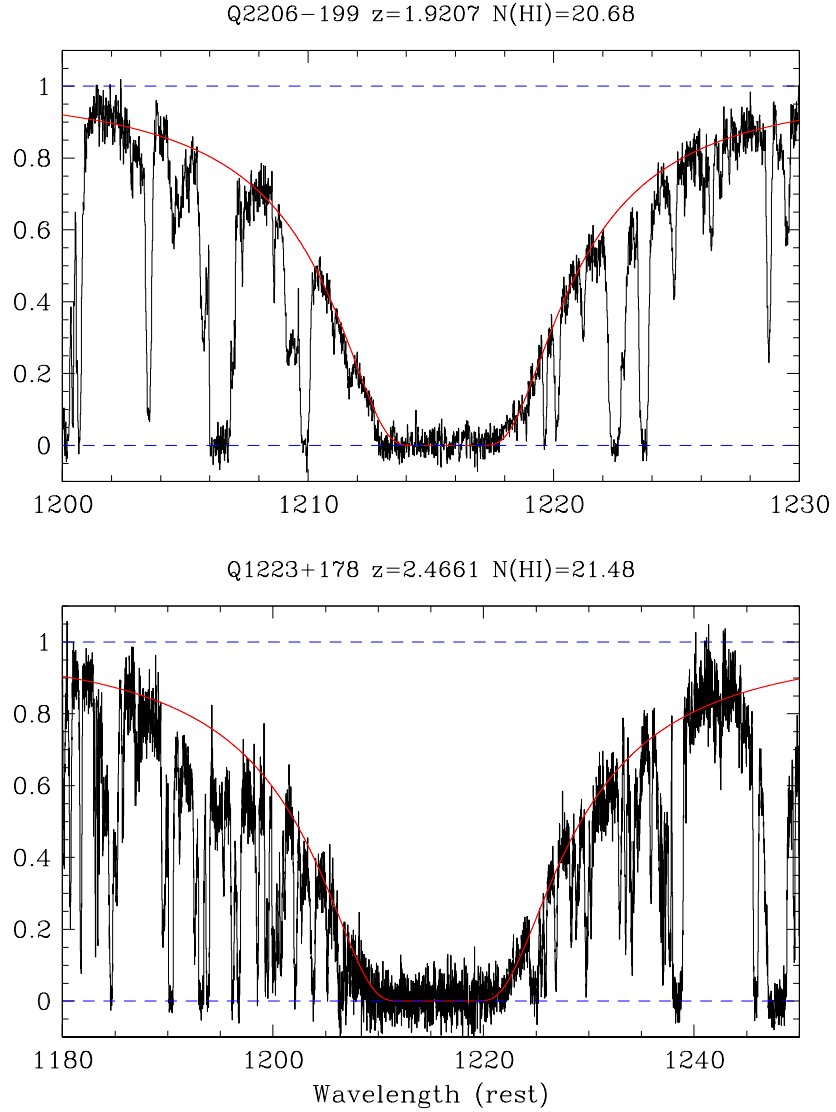

Figure 1. Fits to the damped Lyman $\alpha$ lines for the two QSOs studied here. The data are shown in the rest wavelength for the absorption redshift stated in each panel.

was implemented providing $\mathrm{FWHM} \approx 8 \mathrm{~km} \mathrm{~s}^{-1}$ resolution with the setup covering $\lambda \approx 4750-7200 \AA$. The data were reduced with the MAKEE software package developed by $\mathrm{T}$. Barlow for the extraction of HIRES spectra. The coadded spectrum has $\mathrm{S} / \mathrm{N} \approx 30$ per $2 \mathrm{~km} \mathrm{~s}^{-1}$ pixel.

To obtain the strictest upper limit on the Co II column density for the damped Lyman $\alpha$ system towards Q1223+17, we coadded the HIRES and UVES spectra in the region spanning the Co II $\lambda 2012$ transition. Each spectrum was rebinned to a common wavelength scale and then optimally coadded resulting in a final spectrum with $\mathrm{S} / \mathrm{N} \approx 36$ per 2 $\mathrm{km} \mathrm{s}^{-1}$ pixel. 


\section{NEUTRAL HYDROGEN AND COBALT COLUMN DENSITIES}

The H I column density of the two QSOs was determined by fitting fully damped profiles to the normalised spectra using the Starlink package Dipso. The UVES spectrum of Q2206-199 was found to have a best fit $N(\mathrm{H} \mathrm{I})=4.8 \pm 0.3 \times$ $10^{20} \mathrm{~cm}^{-2}$ and a redshift of $z_{a b s}=1.9207$, Figure 1 . Note that this fit is very well constrained by the shape of the central trough and damping wings and the $N(\mathrm{H} \mathrm{I})$ is in good agreement with previously determined estimates (e.g. Pettini et al. 1994) for this DLA. The HIRES spectrum of Q1223+17 exhibits a relatively large column density of H I which is found to have a best fit of $N(\mathrm{H} \mathrm{I})=3 \pm 0.3 \times 10^{21}$ $\mathrm{cm}^{-2}$ and a redshift of $z_{a b s}=2.4661$, also in good agreement with the Pettini et al. (1994) measurement.

A detailed analysis of other metal abundances in the DLAs towards Q2206-199 and Q1223+17 can be found in Prochaska and Wolfe (1997) and Prochaska et al. (2001); here we simply concentrate on the detection of Co II. However, the analysis of other metal lines plays an important role in determining whether lines are blended and distinguishing weak features from the noise. One of the standard procedures is to fit Voigt profiles to the absorption system which is described as a complex of 'clouds', each defined by a Doppler parameter ( $b$-value), column density $(N)$ and redshift. It has been unanimously found in other high resolution DLA studies that singly ionized species such as Fe II, Si II and Ni II can be fitted using identical cloud models (e.g. Pettini et al 1999). Therefore, line-fitting is often performed first for relatively strong lines with several observed transitions, such as Fe II, in order to fix the $b$-values and redshifts of the constituent components. For the other species, excellent profile fits can be achieved by allowing only the column density to vary as a free parameter, indicating that the singly ionized metal lines have similar kinematical structure. Fixing the cloud model therefore offers a significant advantage when fitting weak lines, especially if there is a risk of some contaminating absorption from other species at a different redshift, e.g. contamination in the Ly $\alpha$ forest.

\subsection{Detection Of Cobalt in the DLA Towards Q2206-199}

In the case of Q2206-199, the absorption system is quite complex in comparison with other DLA metal line systems. The fit was initially constrained using Fe II $\lambda 2249$ and Fe II $\lambda 2260$ and refined by performing a simultaneous fit with Ni II $\lambda 1750$, Ni II $\lambda 1741$ and Ni II $\lambda 1709$. Together, these five transitions can provide excellent constraints on the cloud model and, in addition, are two elements that when considered along with Co may provide interesting clues to nucleosynthetic processes (see section 5). The $b$-values and redshifts of each cloud were tied within the fitting procedure such that these parameters could vary, but remained identical for the two elements. The best fit results for this procedure yielded a 6 component cloud model which extends over almost $150 \mathrm{~km} \mathrm{~s}^{-1}$, see Table 2 . Having determined the cloud model for Fe II and Ni II, we use this template to fit Co II (and the other metal lines), again allowing only the column densities to vary.

The revised $f$-values of Co (Mullman et al. 1998a,b)
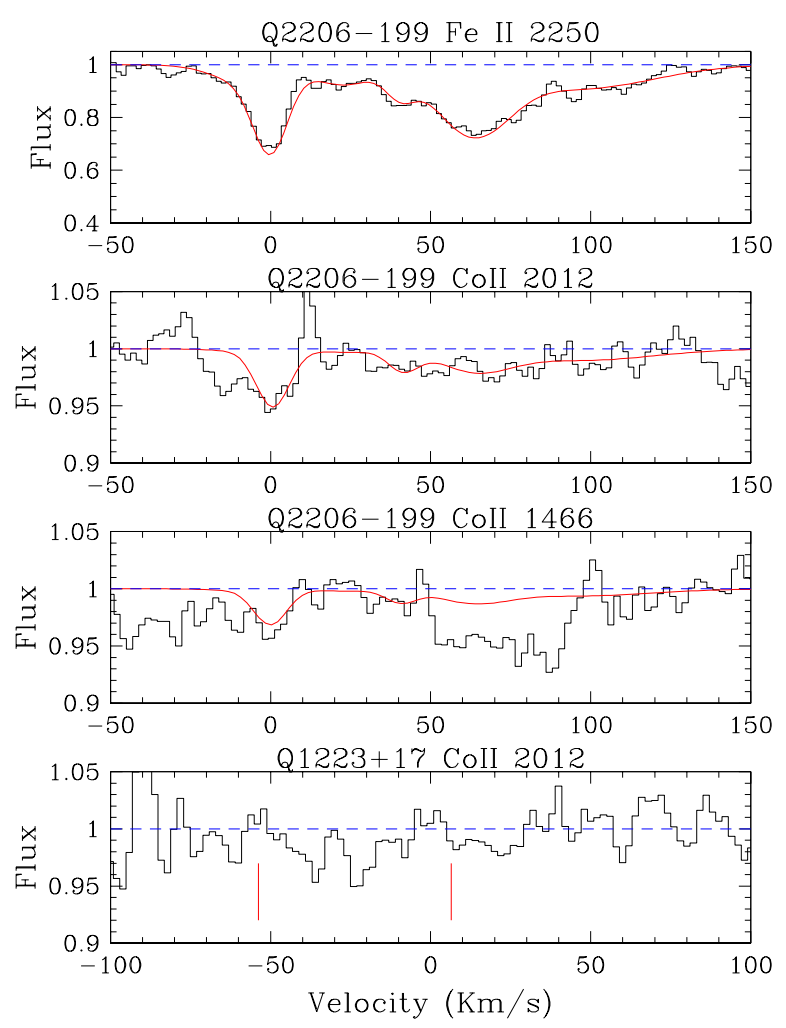

Figure 2. Fits to Fe II $\lambda 2250$ and the two Co II lines observed towards Q2206-199 and the non-detection towards Q1223+17. In all four panels, the data are shown by the solid histogram and the continuum level by the dashed line. For Q2206-199, the fit is shown by the solid curve, and the vertical tick marks in the bottom panel mark the expected positions of the two strongest components in Q1223+17. The Q2206-199 fit was achieved by using Fe II and Ni II transitions to constrain the cloud model and then allowing $N(\mathrm{Co})$ to vary as a free parameter. The Co II $\lambda 1466$ line is blended in the Lyman $\alpha$ forest and is not used to improve the final estimate of $N(\mathrm{Co})$.

show that the two strongest ground state resonance lines covered by this spectrum are at rest wavelengths of 1466.21 $\AA(f=0.031)$ and $2012.16 \AA(f=0.037)$. A third line of similar strength is located at $\lambda=1941.29 \AA(f=0.034)$ but falls in the gap between the two red arm CCDs. The line at $1466 \AA$ for a system at $z_{a b s}=1.92$ lies in the Lyman $\alpha$ forest of the $z_{e m}=2.559$ QSO. The Co II $\lambda 2012$ line lies redward of the QSO's Lyman $\alpha$ emission where the risk of mis-identification or contamination is much lower.

The $\lambda 2012$ line was used to determine the $N(\mathrm{Co})$ of individual components given in Table 2 which also lists the fits to the other Fe-peak elements covered by our spectrum. Although the column density error for any individual component can be quite significant, particularly for closely-spaced, blended lines, the error on the total $\mathrm{N}(\mathrm{X})$ is usually substantially smaller. Also, the error clearly depends on the wavelength of the transition (since the $\mathrm{S} / \mathrm{N}$ varies along the spectrum) and is much reduced if several transitions are used in the fit. All of these factors are taken into account by VPFIT when determining the total column density errors from the chi-squared reduction. In addition, we consider the effect of the continuum fit on our results, which may be particularly 
Table 2. Voigt Profile Fit Parameters for Fe-Peak Elements in the DLA Towards Q2206-19.

\begin{tabular}{|c|c|c|c|c|c|c|c|}
\hline \multirow[t]{2}{*}{ Cloud } & \multirow[t]{2}{*}{ Redshift } & \multirow[t]{2}{*}{$b$} & \multicolumn{5}{|c|}{$\log _{10} N(X)$} \\
\hline & & & Co II & $\mathrm{Ni} I I$ & Fe II & Zn II & Cr II \\
\hline 1 & 1.91995 & 14.8 & 11.54 & 13.09 & 14.42 & 11.88 & 12.56 \\
\hline 2 & 1.92000 & 5.9 & 12.51 & 13.53 & 14.58 & 12.10 & 12.88 \\
\hline 3 & 1.92023 & 11.0 & 11.81 & 13.17 & 14.22 & 11.90 & 12.42 \\
\hline 4 & 1.92040 & 5.1 & 12.05 & 12.95 & 14.15 & 11.87 & 12.38 \\
\hline 5 & 1.92062 & 14.9 & 12.45 & 13.70 & 14.87 & 12.45 & 13.17 \\
\hline 6 & 1.92094 & 31.9 & 12.61 & 13.69 & 14.79 & 12.41 & 13.06 \\
\hline \multicolumn{3}{|c|}{ Total system $\mathrm{N}(\mathrm{X})$} & $13.09 \pm 0.05$ & $14.23 \pm 0.01$ & $15.36 \pm 0.01$ & $12.95 \pm 0.01$ & $13.63 \pm 0.01$ \\
\hline
\end{tabular}

important for Co since a large fraction of the absorption is associated with relatively broad, shallow features. We firstly note that the continuum fit was done with a low order polynomial across the flat continuum around the CoII $\lambda 2012$ line and that any significant departure from this would require quite a contrived placement. Secondly, VPFIT may adjust the continuum in order to improve the fit, although no change was made in this case. As can be seen in Table 3, the relative amounts of $\mathrm{Co}$ in the red and blue components are in good agreement with the other elements, a sign that at least the shape of the continuum is correct. In terms of a systematic offset, in Figure 2 it can be seen that had the continuum been placed too low, there would be clear evidence of flux above the unity level. If the continuum had been placed too high, this would only increase $N(\mathrm{Co})$, thus reinforcing the observed overabundance of $[\mathrm{Co} / \mathrm{Fe}]$. Nonetheless, we have investigated the possible systematic uncertainty induced by continuum placement around Co II and other elements and determine the effect on derived column densities to be not more than 0.10 dex, and typically significantly less than this.

Over-plotting the fit from Co II $\lambda 2012$ onto the Co II $\lambda 1466$ line (Figure 2) shows that although there is evidence for weak Co II absorption there is considerable blending, probably due to Lyman $\alpha$ forest lines. There is also some possibility of blending in the $\lambda 2012$ transition, since it can be seen in Figure 2 that an additional component is present at the blue edge of the absorption complex that is not fitted by the cloud model we adopt (see the top panel of Figure 2 for a comparison with the Fe II $\lambda 2250$ line). In order to investigate the possible impact of this component on our column density determination, we consider the relative contributions of the individual clouds to the total absorption seen in the other metal lines. Table 3 shows the column density of metal enriched gas in the blue $\left(N(X)_{b}\right)$ and red $\left(N(X)_{r}\right)$ components, where the blue component is defined to be made up of clouds 1 and 2 from Table 2, whereas the red component is taken to consist of clouds $3-6$. Also listed in Table 3 is the fraction of gas in the blue component $\left(f_{b}\right)$ for all of the metals measured in this DLA. From these statistics it can be seen that, excluding Co, on average $27 \%$ of the enriched gas resides in the blue component, a value which remains remarkably constant for all the ions considered here. For Co, however, we see that a slightly higher fraction of gas, $29 \%$, resides in the blue component. This small increase may be caused by the wing of the contaminating line. We attempted to identify the possible cause of contamination, but were unable to associate the extra component with any metal line from either of the other 2 DLAs. To show that the effect of
Table 3. Column Densities in the Blue and Red Components of the DLA Towards Q2206-199

\begin{tabular}{cccc}
\hline \hline Element, $X$ & $\log N(X)_{b}$ & $\log N(X)_{r}$ & $f_{b}$ \\
\hline $\mathrm{Co}$ & 12.55 & 12.94 & 0.29 \\
$\mathrm{Fe}$ & 14.81 & 15.22 & 0.28 \\
$\mathrm{Ni}$ & 13.67 & 14.09 & 0.28 \\
$\mathrm{Zn}$ & 12.31 & 12.84 & 0.23 \\
$\mathrm{Cr}$ & 13.05 & 13.50 & 0.26 \\
$\mathrm{Si}$ & 15.23 & 15.63 & 0.28 \\
\hline
\end{tabular}

the blending is probably negligible, we assume that the fractional distribution of gas is the same for $\mathrm{Co}$ as for the other metals and that therefore the $N_{r}(\mathrm{Co})=12.94$ represents $73 \%$ of the total, implying a total $N(\mathrm{Co})=13.08$, almost exactly what is determined from the original fit.

\subsection{The Upper Limit of Cobalt in the DLA Towards Q1223+17}

There is no Co II $\lambda 2012$ absorption visible in the combined UVES+HIRES spectrum of Q1223+17, see Figure 2. To place an upper limit on the $N$ (Co II) value and in turn the $\mathrm{Co} / \mathrm{Fe}$ ratio, we integrated the apparent column density over a small region covering the Co II $\lambda 2012$ transition. For high $\mathrm{S} / \mathrm{N}$ data (i.e. in the limit that $\ln (1-x) \approx x)$, this is equivalent to deriving the equivalent width over the same velocity range and then calculating the column density assuming the linear curve of growth. Although the stronger metal line transitions span over $200 \mathrm{~km} \mathrm{~s}^{-1}$ (e.g. Fe II $1608)$, to place the tightest constraints on the $\mathrm{Co} / \mathrm{Fe}$ ratio we integrated from $-20 \mathrm{~km} / \mathrm{s}$ to $+20 \mathrm{~km} / \mathrm{s}$ which covers the strongest Fe II feature in the velocity profile. Restricting our analysis to this velocity range, we find $N($ Co II $)<12.63$, $N($ Fe II $)=15.05$ and $[\mathrm{Co} / \mathrm{Fe}]<+0.18$ where the limits are at the $3 \sigma$ level.

$\star N_{a}(v)=m_{e} c \tau_{a}(v) / \pi e^{2} f \lambda$ where $\tau_{a}(v)=\ln \left[I_{i}(v) / I_{a}(v)\right], I_{i}$ and $I_{a}$ are the incident and observed intensity, $f$ is the oscillator strength, and $\lambda$ is the rest wavelength of the transition (Savage \& Sembach 1991). 
S. Ellison et al.

\subsection{Corrections Due to Ionization and Dust?}

It is usually assumed in DLA abundance studies that the column densities of the singly ionized metal species are a good approximation to the total column density of this element, based on observations and theoretical modeling (e.g. Viegas 1995). However, there has been some suggestion (Howk and Sembach 1999; Izotov et al. 2000) that ionization corrections may in fact be important when deriving DLA abundances. Although there is some temptation to invoke ionization corrections to explain a few unusual abundance trends in DLAs, the predictions of these models are often not borne out by observations (Pettini et al. 2000; Lattanzio et al 2001). In addition, Levshakov et al. (2000) have recently shown that the physical conditions adopted by these models are unrealistic when compared with those observed in local DLA analogs. Here, we are specifically concerned with the relative abundances of Co and Fe. Even in the unlikely event that some correction is relevant to the absolute abundances as suggested by Izotov et al. (2000), the first and second ionization stages of cobalt and iron have very similar potentials (e.g. $7.88 \mathrm{eV}$ and $7.90 \mathrm{eV}$ for Co I and Fe I) so that it is highly unlikely that an ionization correction is required when converting $\mathrm{N}(\mathrm{Co} \mathrm{II})$ and $\mathrm{N}(\mathrm{Fe} \mathrm{II})$ to $[\mathrm{Co} / \mathrm{Fe}]$. The same is true for nickel, since the ionization potential for $\mathrm{Ni} \mathrm{I}$ is $7.64 \mathrm{eV}$.

The presence of dust in DLAs and its tendency to remove a fraction of the total metals from the gas phase has now been well documented (e.g. Pettini et al. 1994; Pettini et al. 1997). This renders the task of determining the true abundances in these systems somewhat uncertain, although several attempts have been made to recover intrinsic abundances by correcting for Galactic depletion patterns (Vladilo 1998; Savaglio 2000). In the case of Co, some of the more interesting Galactic (stellar) abundance patterns are seen not in absolute (e.g. $[\mathrm{Co} / \mathrm{H}]$ ) measurements, but in the values of relative abundances like $[\mathrm{Co} / \mathrm{Fe}]$ and $[\mathrm{Co} / \mathrm{Cr}]$. The important consideration is therefore the relative depletion fractions of these elements, which can be determined from local interstellar observations. From Savage and Sembach (1996) and Mullman et al. (1998b) we can see that Co, Cr, $\mathrm{Ni}$ and $\mathrm{Fe}$ are all depleted to a very similar level. In addition, at relatively low levels of depletion, as is the case for Q2206-199, the small differences between the dust correction required for these elements becomes negligible (Pettini et al. 2000).

Therefore, we assume that we can safely investigate relative abundance ratios such as $[\mathrm{Co} / \mathrm{Fe}]$ without the need for either corrections for dust or ionization.

\section{DISCUSSION OF COBALT ABUNDANCES}

In Table 4, we list the abundances of the iron peak elements (Fe, Ni, Cr, Zn, Co) measured for the two DLAs analysed herein and two other systems found in the literature for which useful $[\mathrm{Co} / \mathrm{H}]$ upper limits can be determined.

\subsection{Homogeneity of Stellar Cobalt Abundances}

The cobalt abundances measured in the four DLAs will be compared in Section 5.2 with those for stars spanning a range of Galactic populations and metallicities (Sneden \& Gratton 1991; McWilliam \& Rich 1994; McWilliam et al. 1995; Ryan, Norris, \& Beers 1996; Prochaska et al. 2000). The stellar measurements use solely Co I lines.

All stellar measurements utilised laboratory $g f$ values from Cardon et al. (1982), with the exception of Sneden \& Gratton who derived solar $g f$ values. These two scales appear to be very similar; five lines in common give a mean difference $\log g f_{\mathrm{GS} 90}-\log g f_{\mathrm{CSSTW}} \mathrm{C2}=+0.06$ dex with a standard error in the mean of 0.04 dex. As this is significant only at the $1.5 \sigma$ level, it would be premature to adjust for it. Furthermore, by using solar $g f$ values, Sneden and Gratton have effectively analysed their stars differentially with respect to the Sun, so changing the $g f$ values would alter the inferred solar abundance as well. We note, nevertheless, that even in their most metal-rich stars, $[\mathrm{Co} / \mathrm{Fe}]$ tends towards $\simeq-0.05$ at $[\mathrm{Fe} / \mathrm{H}]=0$, which supports the view that their Co abundances are marginally low. The problem (assuming there is one) may perhaps be traced to differences in the assumptions in the solar model used for their solar analysis and those employed in the other stellar calculations. We did, however, correct the three stars of Sneden \& Gratton affected by the probable 0.47 dex error in the solar log $g f$ value of the $4118 \AA$ line; see Norris, Ryan \& Beers (2001) for details. A comparison with the improved $g f$ values of Nitz et al. (1999) confirms the accuracy of the Cardon et al. scale: $\log g f_{\mathrm{CSSTW}} 82-\log g f_{\mathrm{NKWL}}{ }_{29}=-0.02$ dex and $\sigma_{\mathrm{CSSTW}} 82$ $\simeq 0.08$ dex. We are thus confident that all stars are on a uniform, accurate $g f$ scale. This is quite an achievement since the stars span four orders of magnitude in abundance, and have been analysed by several independent groups.

As the DLA Co abundances are derived from ionised lines whereas the stellar transitions are neutral, there is (in principle) a possibility that the stellar and DLA abundance scales differ. However, as the $g f$ scales for both ionisation states are tied to modern lifetime measurements, we doubt that differences exceed $5-10 \%$, or $0.02-0.04$ dex.

Co I lines have extensive hyperfine structure which can affect abundance measurements by up to several $\times 0.1$ dex (e.g. Ryan et al. 1996, Fig. 1). The stellar studies cited have all accounted for hyperfine structure, which was not always the case in earlier analyses. Moreover, work by Pickering and colleagues (1998 and priv.comm.) have confirmed that the Co II lines we have observed in the DLAs will not be broadened sufficiently to affect our results.

Both Co and Fe are primarily singly ionised in the temperature range of the stars investigated, so the Co I measurements are of a minority state. However, because these elements have almost identical first ionisation potentials, uncertainties in their temperatures or surface gravities will affect the ionisation degree of Co I just as much as for Fe I. Representative errors in $[\mathrm{Co} / \mathrm{Fe}]$ - see Ryan et al. 1996, Table 3 - appear to be $\simeq 0.05-0.10$ dex $(1 \sigma)$ for $\operatorname{good} \mathrm{S} / \mathrm{N}$ $(\sim 100)$ data, but can be $\sim 0.2$ dex for poorer data with $\mathrm{S} / \mathrm{N}$ $\simeq 30$. It is believed that the lower $\mathrm{S} / \mathrm{N}$ levels achieved in the first studies of the most metal-poor stars may explain most of the spread in $[\mathrm{Co} / \mathrm{Fe}]$ seen in those stars. (See Norris et al. 2001 for much improved $[\mathrm{Co} / \mathrm{Fe}]$ measurements in such objects.) 
Table 4. Iron-peak abundances. Co limits are $3 \sigma$. Note that the Ni abundance for HE1104-1805 has been increased by a factor of 1.9 from the published value, based on the revised $f$-values of Fedchak and Lawler (1999)

\begin{tabular}{lcccccl}
\hline \hline Quasar & {$[\mathrm{Fe} / \mathrm{H}]$} & {$[\mathrm{Ni} / \mathrm{H}]$} & {$[\mathrm{Co} / \mathrm{H}]$} & {$[\mathrm{Cr} / \mathrm{H}]$} & {$[\mathrm{Zn} / \mathrm{H}]$} & Ref \\
\hline Q2206-199 & -0.81 & -0.70 & -0.50 & -0.73 & -0.42 & This work \\
Q1223+17 & -1.94 & -1.80 & $<-1.76$ & -1.66 & -1.94 & Prochaska et al. (2001), this work \\
Q0302-223 & -1.20 & -1.04 & $<-0.87$ & -0.96 & -0.56 & Pettini et al. (2000) \\
HE1104-1805 & -1.59 & -1.40 & $<-1.56$ & -1.46 & -1.02 & Lopez et al. (1999) \\
\hline
\end{tabular}

\subsection{Comparison with Relative Stellar Abundances}

The stellar data discussed in the previous section are plotted in Figure 3, as a function of $[\mathrm{Fe} / \mathrm{H}]$. As discussed in the Introduction, Galactic stellar $[\mathrm{Co} / \mathrm{Fe}]$ ratios show distinct — but non-monotonic - trends as a function of metallicity, which indicate key differences between the various stellar populations. The halo and bulge data, for example, at high and low metallicity, share great age and the highest $[\mathrm{Co} / \mathrm{Fe}]$ ratios, whereas later-forming stars in the disk have progressively lower $[\mathrm{Co} / \mathrm{Fe}]$ ratios. In addition, the Co detection in Q2206-199 and three upper limits are also plotted, although for the DLAs $[\mathrm{Zn} / \mathrm{H}]$ is used as a metallicity indicator rather than $[\mathrm{Fe} / \mathrm{H}]$. The first determination of $[\mathrm{Co} / \mathrm{Fe}]=$ $+0.31 \pm 0.05$ in a DLA is consistent with the overabundance seen in Galactic halo stars and the transition regime between the thick disk and bulge at a metallicity consistent with the latter populations. The point plotted for Q2206-199 is the value determined from our Voigt profile fit although, as discussed in section 4 , this may be a small overestimate if there is some blending of the blue components. In addition, Co is also overabundant relative to $\mathrm{Cr}$ in Q2206-199, [Co/Cr] = +0.23 , again consistent with thick disk, moderately metal poor halo and metal-rich bulge stars.

The trend of high $[\mathrm{Co} / \mathrm{Fe}]$ in metal-poor stars was first pointed out by McWilliam et al. (1995) and confirmed by Ryan et al. (1996). The observation that stellar $[\mathrm{Co} / \mathrm{Fe}]$ abundances are highest in the oldest populations might suggest that elevated abundances arise from the fastest evolving stars, i.e. the most massive supernova (SN) progenitors. However, halo star $[\mathrm{Co} / \mathrm{Fe}]$ values are not uniformly high, and clearly vary as a function of metallicity. McWilliam et al. (1995) suspected that the trends were due to a metallicity dependence of the yields of elements produced in supernova (SN) nucleosynthesis, while Ryan et al. (1996) noted that differences in the explosion energy could affect the yields in this way. It is unclear whether the metallicity-dependence is secondary, being driven by lower $\mathrm{Co} / \mathrm{Fe}$ yields from laterevolving, lower mass $\mathrm{SN}$, or whether it reflects an explicit dependence on progenitor metallicity. More than one factor may be (and probably is) at work. Umeda et al. (2000) have shown that the abundances of these elements do not depend strongly on the initial stellar metallicity. Nakamura et al. (1999) have shown that the abundance trends of $\mathrm{Cr}$, Co and Mn observed in the most metal-poor stars can be reproduced when changing the position of the mass cut, but at the expense of overproducing $\mathrm{Ni}$, which is only rarely observed in the stars (Ryan et al. 1996). More recently, Nakamura et al. (2000) have also been able to reproduce the trends, though not the absolute values, in $\mathrm{Co}$ and $\mathrm{Cr}$ by using

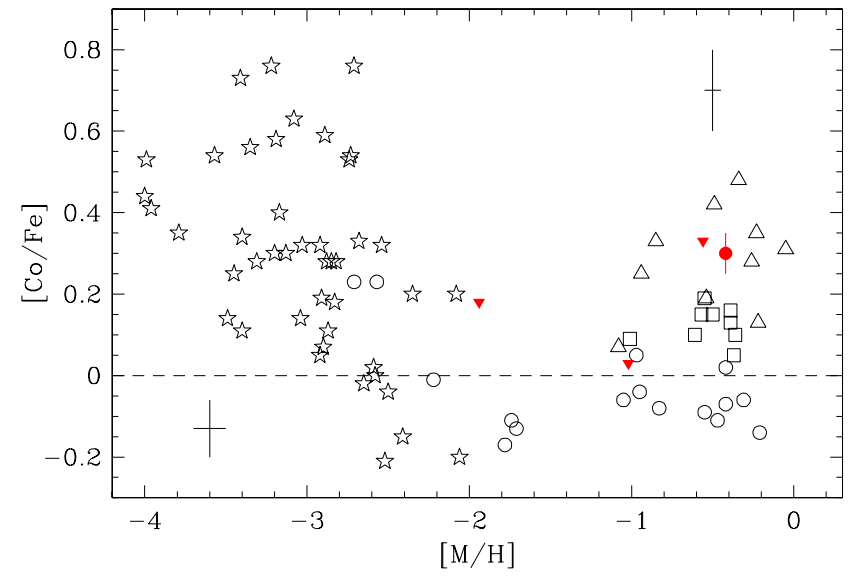

Figure 3. Relative cobalt abundances as a function of metallicity for different Galactic populations and DLAs: open stars - metalpoor halo stars from McWilliam et al (1995) and Ryan, Norris, \& Beers (1996); open circles - moderately metal-poor thin disk and halo stars from Sneden \& Gratton (1991), with $g f$ changes as described in the text; open triangles - bulge stars from McWilliam \& Rich (1994); open squares - thick-disk stars from Prochaska et al. (2000). The solid circle represents Q2206-199 (with associated fitting error) and the down-turned filled triangles are upper limits for three other DLAs. The cross in the lower left corner of the figure shows the typical error bar for stellar points, whereas the error bar in the top right corner shows the maximum effect of systematics (continuum placement and $N(\mathrm{H} \mathrm{I})$ determination) discussed in the text.

nucleosynthesis models of hypernovae, i.e. supernovae with very high explosion energies. These authors remark that as well as re-producing Galactic abundances, they can also explain the unusual patterns seen in some starburst galaxies, such as M82. Indeed, several other observational clues indicate that star formation in DLAs proceeds in short bursts separated by extended quiescent periods, such as low N/O and $[\mathrm{S}, \mathrm{Si} / \mathrm{Zn}]$ values (Pettini et al. 2001; Pettini et al 2000) and the lack of detected star formation through $\mathrm{H} \alpha$ imaging down to levels of $\sim 1 \mathrm{M}_{\odot} \mathrm{yr}^{-1}$ (Bouche et al. 2000; Kulkarni et al. 2001). However, both the models of Nakamura et al. $(1999 ; 2000)$ also predict an overabundance of Ni relative to Fe, something that is rarely seen in stars, but quite commonly seen in DLAs (Figure 4). In fact all 4 of the DLAs plotted in Figure 3 exhibit $[\mathrm{Ni} / \mathrm{Fe}] \sim 0.2$ I. However, since at

$\dagger$ We note that Prochaska \& Wolfe (1997) use the old NiII fvalues, requiring a correction by a factor of 1.9 , which brings their $\mathrm{N}(\mathrm{Ni})$ into very good agreement with ours. 
least HE1104-1805 shows no strong overabundance of Co, it appears that neither stars nor DLAs exhibit consistent or comprehended relative abundances of Co and Ni. Finally, we note that since the revision of Ni II $f$-values, a systematic overabundance is now seen for [Ni/Fe] in DLAs, perhaps indicating that further refinement of the atomic data may still be required.

As detailed supernova nucleosynthesis models have yet to provide a convincing explanation of the $[\mathrm{Co} / \mathrm{Fe}]$ abundances of stars, we would not presume to be able to solve that mystery with the first ever DLA observations. Nevertheless, by comparing the DLA data with the stellar measurements, useful insights can be obtained. As noted, the $[\mathrm{Co} / \mathrm{Fe}]$ ratio in the $z_{\mathrm{abs}}=1.92$ DLA of Q2202-199 falls near the boundary between bulge and thick disk stars. It is seen at a lookback time of $\simeq 8.5 \mathrm{Gyr}$, around the time of (or a few Gyr after) the formation of the oldest thick disk and bulge objects. However, the possible absence of an elevated $[\mathrm{Co} / \mathrm{Fe}]$ ratio in $\mathrm{Q} 1223+17$, with a lookback time slightly $(\simeq 0.5 \mathrm{Gyr})$ longer, emphasises that age alone does not explain the elevated $[\mathrm{Co} / \mathrm{Fe}]$ values. If massive stars do produce the elevated $[\mathrm{Co} / \mathrm{Fe}]$ ratios, then possibly star formation started later in the $z=1.92$ DLA than in the others. This postulate could be further explored by considering the abundance of $\mathrm{Si}$, an $\alpha$ element. We find $[\mathrm{Si} / \mathrm{Zn}]=-0.07$, although this value is likely be be an underestimate of the true Si abundance due to dust depletion. It is possible to make a crude adjustment for the depletion fraction by considering $\mathrm{Zn}$ and $\mathrm{Cr}$ ratios, which are found to be solar in stars. Therefore, any significant departure from $[\mathrm{Zn} / \mathrm{Cr}]=$ 0 can be explained by the incorporation of $\mathrm{Cr}$ into grains. For Q2206-199, we determine $[\mathrm{Zn} / \mathrm{Cr}]=0.35$ dex, applying this correction to $\mathrm{Si}$ gives $[\mathrm{Si} / \mathrm{Zn}] \sim 0.3$, although this is likely to be an upper limit since $\mathrm{Si}$ is less depleted than $\mathrm{Cr}$ in the local ISM (Savage and Sembach 1996). In any case, it is likely that $\mathrm{Si}$ exhibits at least a mild overabundance relative to $\mathrm{Zn}$, consistent with the expectation if the enrichment of this galaxy's ISM has been dominated by the products of Type II SN.

Nakamura et al. $(1999,2000)$ have achieved partial success in attempting to explain the iron-group element ratios (Ni excepted) in their nucleosynthesis models, firstly by moving the mass-cut and secondly by changing the explosion energy. The latter, as they note, partially simulates the former effect because the zone of complete Siburning is shifted outward in high-energy models compared to classical $10^{51}$ erg models. However, increasing the energy has the additional beneficial outcome that it provides a solution, for the first time, to the long-standing problem of explaining why $\mathrm{Ti}$ is overabundant in halo stars. It is found that the greater radial extent over which Si-burning occurs in the high-energy models necessarily incorporates lower density zones, which allows for increased $\alpha$-rich freezeout-production of ${ }^{48} \mathrm{Ti}$. Previous classical SN nucleosynthesis models always yielded $[\mathrm{Ti} / \mathrm{Fe}] \simeq 0$. If this finding may be considered independent evidence that higher-energy explosions are the norm in the more massive $\mathrm{SN}$, i.e. those that enriched the extremely metal-poor halo and the 'getrich-quick' bulge, then we should understand the higher $[\mathrm{Co} / \mathrm{Fe}]$ and simultaneously the higher $[\mathrm{Ti} / \mathrm{Fe}]$ ratios in these populations that were enriched by massive-stars. However, Prochaska \& Wolfe (1997) find that $[\mathrm{Ti} / \mathrm{Fe}]=-0.07$ in

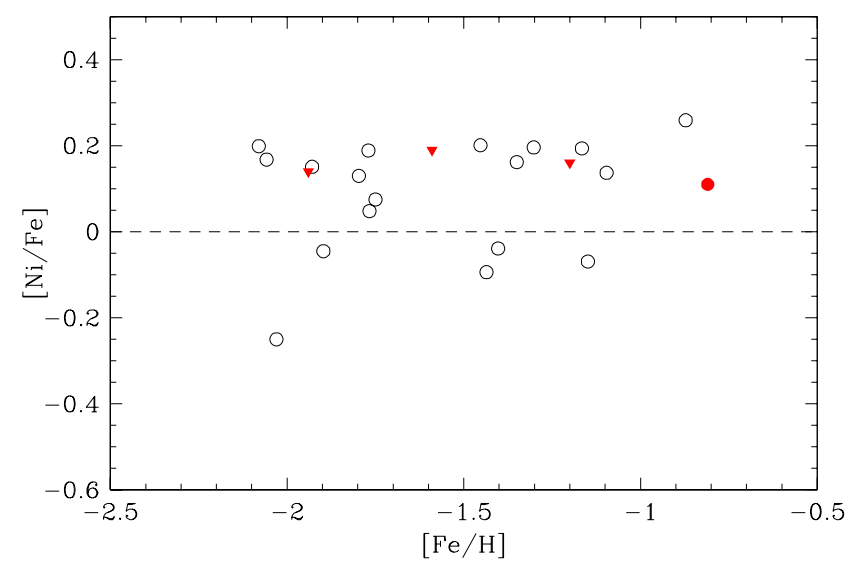

Figure 4. Ni and Fe abundances in DLAs. Open circles are values taken from the literature (Prochaska et al 2001; Pettini et al. 2000), filled triangles are the DLAs for which a Co upper limit has been obtained herein and the filled circle is for the DLA towards Q2206-19 for which Co has been detected.

Q2206-199, although considering that $\mathrm{Ti}$ is slightly more depleted than Fe (Savage and Sembach 1996), this DLA is likely to have a roughly solar $\mathrm{Ti} / \mathrm{Fe}$ ratio.

Once high-energy SN models are explored more widely and included into galactic chemical evolution calculations, and a larger range of elements including Co and possibly $\mathrm{Ti}$ have been measured in more DLAs, the complementary constraints presented by DLA and stellar systems can be considered jointly. Nevertheless, it is important to appreciate through all of this that the similarities of DLA $[\mathrm{Co} / \mathrm{Fe}]$ and $[\mathrm{Co} / \mathrm{Cr}]$ ratios to those of a given Galactic population do not necessarily imply that this DLA, or the DLA population in general, represents the precursor to modern day halos/bulges. Rather, it is indicative of similar physical properties such as star formation rate and mix of SN types between the populations.

\section{SUMMARY AND CONCLUSIONS}

The distinct trends exhibited by Co in the various Galactic stellar populations makes this an interesting element to target in DLA abundance studies. However, the weakness of the Co II lines renders this a challenging prospect, even for an 8-m class telescope such as the VLT. Nonetheless, we have shown that by selecting DLAs with high column densities of Fe towards bright QSOs, the detection of Co is feasible with instruments such as UVES and HIRES down to $[\mathrm{Co} / \mathrm{Fe}] \sim$ 0 . We have achieved the first detection of $[\mathrm{Co} / \mathrm{H}]=-0.50$ in one of the DLAs studied herein and a sensitive upper limit for a second system. In addition, we have determined useful upper limits for $[\mathrm{Co} / \mathrm{H}]$ for two other DLAs from the literature. The detection of Co towards Q2206-199 shows an overabundance relative to $\mathrm{Fe},[\mathrm{Co} / \mathrm{Fe}]=+0.31 \pm 0.05$, at a metallicity of approximately $\frac{1}{3} Z_{\odot}$, consistent with both the ratios observed in Galactic bulge and thick disk stars. In fact, Prochaska et al (2000) noted that there is evidence from Galactic bulge/thick disk stellar abundances that these two populations may have shared a similar formation history. One current limitation of the comparison with bulge stars, however, is that the only measurements presently available 
in the literature were obtained with a 4-m telescope with relatively low $(R \sim 17,000)$ resolution (McWilliam and Rich 1994). Repeating these observations with Keck/HIRES has shown that these abundances may undergo significant revision, for example $[\mathrm{Fe} / \mathrm{H}]$ has already been found to be higher by $\sim 0.2$ compared with the $4-\mathrm{m}$ data of lower resolution and S/N (Rich and McWilliam 2000). The revised Co abundances from the HIRES data are not yet available, but will eventually provide an interesting comparison for our DLA detection. Conversely, the upper limit of $[\mathrm{Co} / \mathrm{Fe}] \leq+0.18$ that we determine for Q1223+17 is not consistent with the bulge star pattern and is more in line with Galactic disk abundances, as is the result for HE1104-1805.

However, the interpretation of the data presented here are limited by statistics and we clearly can not presume to understand the star formation history of DLAs based on a single detection. In addition, Q2206-199 is already known to exhibit large abundances of other elements which are not normally seen in DLAs, such as Ti (Prochaska and Wolfe 1997). We can therefore not exclude the possibility that Q2206-199 exhibits peculiar abundances that may not be seen in other such systems. Finally, we have deliberately chosen DLAs with high $N(\mathrm{Fe})$ for this initial study in order to maximise the probability of a Co detection. In order to extend this analysis, such a selection bias will eventually have to be overcome if we are to truly understand the trends of relative abundances. Nonetheless, this is an interesting new result and the study of Co in DLAs clearly invites further study.

Our understanding of the processes that drive abundance trends in stars has only truly become possible thanks to large datasets, since intrinsic scatter and errors will dominate small samples. Therefore, more DLA data points are clearly required in order to draw firm conclusions about the star formation histories impliat by the trends. Perhaps most importantly, we have shown that with current 8-m class telescopes and high resolution echelle spectrographs that such measurements are not only possible, but also that Co may be a rewarding element to study in the context of testing nuclesynthetic models and understanding galactic star formation histories.

\section{ACKNOWLEDGMENTS}

We are very grateful to our collaborators Max Pettini, Jacqueline Bergeron and Patrick Petitjean for consenting to release the data for Q2206-199, originally obtained for a different project. It is a pleasure to acknowledge the staff of the VLT and Keck for their assistance during the acquisition of these data. In particular we are grateful to Andreas Kaufer for his support during both the VLT observations and data reduction. We would like to thank Chris Howk and Emmanuel Jehin for helpful discussions and to Max Pettini and Norbert Christlieb for useful comments on an earlier draft. We are grateful to Sebastian Lopez for providing his Co upper limit in the DLA towards HE1104-1805.

\section{REFERENCES}

Ballester, P., Modigliani, A., Boitquin, O., Cristiani, S., Hanuschik, R., Kaufer, A., Wolf, S., 2000, ESO Messenger,
101, 31

Bouche, N., Lowenthal, J., Charlton, C., Bershadv, M. Churchill, C., Steidel, C., 2000, ApJ, accepted, astro-ph/0011374

Cardon, B. L., Smith, P. L., Scalo, J. M., Testerman, L., \& Whaling, W. 1982, ApJ, 260, 395

Centurión, M., Bonifacio, P., Molaro, P., \& Vladilo, G. 2000, ApJ, 536,540

Chen, Y.Q., Nissen,P.E., Zhao, G., Zhang, H.W., \& Benoni, T. 2000, A\&AS, 141, 491

Ellison, S. L., Yan, L., Hook, I., Pettini, M., Shaver, P., Wall, J., 2000, ESO Messenger, 102

Fedchak, J. A., \& Lawler, J. E. 1999, ApJ, 523, 734

Federman, S., Sheffer, Y., Lambert, D., Gilliland, R., 1993, ApJl, 413,51

Gratton, R., \& Sneden, C., 1991, A\&A, 241, 541

Howk, J.C., \& Sembach, K.R. 1999, ApJ, 523, L141

Izotov, Y.. Schaerer, D., Charbonnel, C., 2000, ApJ, accepted, astro-ph/0010643

Izotov, Y. I. \& Thuan, T. X., 1999, ApJ, 511, 639

Kobulnicky, H.A., \& Zaritsky, D. 1999, ApJ, 511, 118

Kulkarni, V., Hill, J., Schneider, G., Weymann, R., StorrieLombardi, L., Rieke, M.. Thompson, R., Jannuzzi, B., 2000, ApJ, accepted, astro-ph/0012140

Lattanzio, J., Pettini, M., Tout, C. A., \& Carigi, L. 2000, A\&A, submitted

Levshakov, S., Kegel, W., Agafonova, I., 2000, A\&A, submitted astro-ph/0011513

Lopez, S., Reimers, D., Rauch, M., Sargent, W., Smette, A., 1999, ApJ, 513, 598

Lu, L., Sargent, W. L. W., Barlow, T.A. 1998, AJ, 115, 55

McWilliam, A., \& Rich, M., 1994, ApJS, 91, 749

McWilliam, A., Preston, G. W., Sneden, C., Searle, L., 1995, AJ, 109,2757

Mullman, K., Cooper, J., Lawler, 1998, ApJ, 495, 503

Mullman, K., Lawler, J., Zsargo, J., Federman, S., 1998, ApJ, 500,1064

Nakamura, T., Umeda, H., Iwamoto, K., Nomoto, K., Hashimoto, M.. Hix. R , Thielemann, F., 2000, ApJ, submitted, astro$\mathrm{ph} / 0011184$

Nakamura, T., Umeda, H., Nomoto, K., Thielemann, F., Burrows, A., 1999, ApJ, 517, 193

Nitz, D. E., Kunau, A. E., Wilson, K. L., \& Lentz, L. R. 1999, ApJS, 122, 557

Norris, J. E., Ryan, S. G., \& Beers, T. C. 2001, in prep.

Pettini, M., Ellison, S. L., Steidel, C. C., Bowen, D. V. 1999, ApJ, 510,576

Pettini, M., Ellison, S. L., Steidel, C. C., Shapley, A. E., \& Bowen, D. V. 2000, ApJ, 532, 65

Pettini, M., et. al 2001, in preparation

Pettini, M., Smith, L.J., King, D.L., \& Hunstead, R.W. 1997, ApJ, 486, 665

Pettini, M., Smith, L.J., Hunstead, R.W., King, D.L., 1994, ApJ, 426,79

Pickering, J. C., Raassen, A. J. J., Uylings, P. H. M., \& Johansson, S. 1998, ApJS, 117, 261

Prantzos, N., Boissier, S., 2000, MNRAS, 315, 82

Prochaska, J. X., et al., 2001, in preparation.

Prochaska, J. X., Naumov, S.O., Carney, B.W., McWilliam, A., \& Wolfe, A. M. 2000, ApJ, accepted

Prochaska, J. X., \& Wolfe, A. M. 1997, ApJ, 474, 140

Prochaska, J.X., \& Wolfe, A.M. 1999, ApJS, 121, 369

Rich, M., \& McWilliam. A., 2000, Proceedings of the SPIE vol. 4005, astro-ph/0005113

Ryan, S. G., Norris, J. E., \& Beers, T. C., 1996, ApJ, 471, 254

Savage, B. D. \& Sembach, K. R., 1996, ARA\&A, 34, 279

Savage, B. D. \& Sembach, K. R., 1991, ApJ, 379, 245

Savaglio, S., 2000, Invited talk at the IAU Symposium 204 'The Extragalactic Infrared Background and its Cosmological Im- 
plications', Manchester, August 2000, eds. M. Harwit \& M.G. Hauser, astro-ph/0011473

Shetrone, M.. Cote, P., Sargent, W. L. W., 2000, ApJ accepted, astro-ph/0009505

Tinsley, B. M. 1979, ApJ, 229, 1046

Umeda, H., Nomoto, K., Nakamura, T., 2000, in The First Stars, pp. 150-174 astro-ph/9912248)

Viegas, S.M. 1995, MNRAS, 276, 268

Vladilo, G., 1998, ApJ, 493, 583

(C) 2001 RAS, MNRAS 000, 10 